\title{
Innovation Management in Educational Technology
}

\author{
Xiaohui Zou ${ }^{1,2, *}$ \\ ${ }^{1}$ Sino-American Searle Research Center, Zhuhai Hengqin Searle Technology Co., Ltd., Guangdong 519000, China \\ ${ }^{2}$ Xiaoqun Wang Research Group, Teacher Teaching Development Center, Peking University, Beijing 100871, China \\ *Corresponding author.Email: 949309225@qq.com
}

\begin{abstract}
\section{INTRODUCTION}

The first mission and basic function of the university is teaching.There are many teaching reforms around $\mathrm{e}$ schools and e-universities, but it is unique to be able to do quality assurance in educational technology from the perspective of China IOT names service platform Smart System Studied Application Promotion Center. [1-8]

The informatization of education and teaching has been conducted for many years, and it turns out that people are difficult to control. However, there are a series of key objects in the integration of teachers and students, and they are relatively easy to master. This is an entry point for this topic to base itself on the identity of the Internet of Things.
\end{abstract}

The aim is to explore the entry point for this topic to base itself on the identity of the Internet of Things machine-assisted by the first-level node and the second-level node. The method is China Internet of Things names management public service platform Smart System Studied Application Promotion Center connects them in the way of digitization, networking, informationization, knowledge and intelligence, and achieves a significant improvement in the social system engineering for teaching, management, learning and using. Its characteristic lies in the purpose of quality. The result is China Internet of Things names analysis example for more than 7,000 courses in Peking University is not only a demonstration model of a unified names analysis system for basic carrier objects, but also corresponding classrooms. Its significance lies in the further improvement and optimization of the five of digitization, networking, informationization, knowledge and intelligence that the advantages of human-machine collaborative smart system are easier to form.

Keywords: Innovation management in educational technology; Quality assurance in educational technology;

Educational technology resources and funding; Web-based education and training; Educational network

management;Paperless practice system;Paperless examination system;E-school and e-university

\section{PROBLEMS AND METHODOLOGY}

\subsection{Problems and Hypothesis}

The issues that this research focuses on and the basic assumptions it put forward are:

Whether it is a textbook, or an electronic textbook or courseware (PPT) or even a high-quality course (audio and video as rich media), they all belong to the basic category of things as carriers. This brings up several basic questions of this research: In the era of digitization, networking, information, knowledge and intelligence (modernizations as five ones), they can all become the basic objects of the
Internet of Things name and its analysis. Therefore, typical Internet of Things names such as barcodes and later QR codes, color codes, graphic codes, etc., are essentially the basic category of textual information, which is in contrast to the Eight Major Forms System (natural language $\mathrm{Zi}$ and word,formula,graphic,tables,audio,visual,three-dimensional and livings) are all linked together. At this point, the carrier (thing) with space-time quality and energy has overlapped with the form information (text) and content information (meaning the basic category of choice intentions) with order and information. The biggest ambiguity problem then arises. Therefore, the basic hypothesis of this research is: the previous extensive knowledge processing methods (textbooks, courseware, high-quality courses as rich media by using audio and video) must be upgraded to finegrained pages, examples and ontology, otherwise, it will be difficult to achieve the expected results of sustainably improving the quality of teaching or beneficial effects.

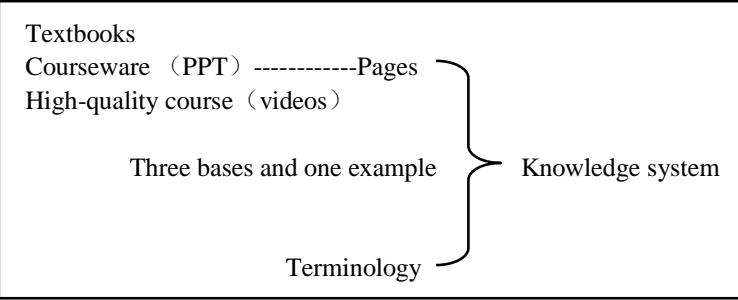

Figure 1. Schematic diagram of the relationship between the extensive and fine processing methods 
It can be seen from Figure 1 that the pages based on the existing textbooks, courseware and videos are related to basic concepts, basic principles, basic methods, and typical examples (three bases and one example) and their term ontology (knowledge ontology). They are combined to form the knowledge system of various disciplines.

The most important thing is that based on the page (three bases and one case of term ontology), creativity, creation, creation, innovation and entrepreneurship ("five creations") can be done well in the finishing of knowledge modules. This can very well support our basic hypothesis to be verified, thus paving the way for the traditional small-scale production of personal knowledge and the large-scale production of computer-aided knowledge modules.

\subsection{Research Method and Main Process}

We know that the university's logistics materials, especially fixed assets, are accounted for, involving educational technology resources and funds. However, they are not mutually exclusive with the curriculum arrangement of the Academic Affairs Office and the arrangement of multimedia tools in the audio-visual department. Related, there is almost no direct connection with the commonly used teaching aids, learning aids, appliances, tools, and other appliances used by teachers and students. The National Internet of Things logo management public service platform Smart System Studied application promotion center connects them in the way of digitization, networking, informationization, knowledge and intelligence, and achieves a significant improvement in the social system engineering for teaching, management, learning and using. Practice the purpose of quality.

The basic relationship between teachers and students is equivalent to the relationship between master and apprentice. Simply put: the relationship between experienced teachers and uninitiated laymen. The most typical is the process of compiling teaching materials, making teaching plans, and teaching excellent courses. Under the conditions of education informatization, the process of continuously improving the quality of distance teaching with the help of "five modernizations" technology. Not only students but also other teachers must study and digest them seriously. To this end, this research proposes a basic method for further deep processing: first, at the carrier level, pageization; then, at the level of knowledge intention, highlighting the three bases and one example; finally, at the level of language as text, focusing on terminology ontology. On the whole, it highlights a complete system of macro and micro links in teaching and management. And work hard on the actual mastery of language points, knowledge points and original points ("three points"). Not only play the role of every experienced teacher, but also play the role of computer Internet of Things logo and its analysis in a timely manner (provide computer-assisted teaching cases for each subsequent teacher and student user).

\section{RESULTS, ANALYSIS AND DISCUSSION}

The innovative management of educational information technology from textbooks to courseware and then to quality courses, focuses on language points, knowledge points and original points, these three points can be recorded on the page (any one of the three bases and one example). Each page has a unique IoT logo or name. The three bases are: basic concepts, basic principles, and basic methods; one example is a typical example.

\subsection{Results: Focusing on the three basics and an example in the form of pages}

The result is focusing on the three basics and an example in the form of pages. For example, China Internet of Things names analysis example for more than 7,000 courses in Peking University is not only a demonstration model of a unified names analysis system for basic carrier objects such as textbooks, courseware, and video and audio records, but also demonstration model of unified identification analysis system for various teaching aids, such as blackboards, projectors, screens, and even experimental instruments and corresponding classrooms, desks, and benches. This result seems to be relatively simple to operate, but in fact it is very effective in terms of big data management.

Table 1. Application examples of new generation information processing technology

\begin{tabular}{|c|c|c|c|}
\hline \multirow{2}{*}{ Digit } & \multicolumn{3}{|c|}{ Three essentials of information processing } \\
\cline { 2 - 4 } & Carrier & Content & Form \\
\hline 001 & Textbooks $^{\text {a }}$ & Edited by experts & Forms \\
\hline 002 & Courseware $^{\text {b }}$ & 3-Bases, 1-example & Pages \\
\hline 003 & Videos $^{2}$ & Experts teaching & Forms \\
\hline \multicolumn{4}{|c|}{${ }^{\text {a. }}$ Textbooks. $^{\text {b. }}$ PPT } \\
\hline
\end{tabular}

It can be seen from Table 1 that the application examples of the new generation of information processing technology cover the three essentials of information processing: information carrier, information content and information form. Among them, three types of information carriers: textbooks, textbooks, courseware, and quality lessons or videos, are the basic objects of the Internet of Things names and its analysis. These are the ones that the computer can directly participate in the analysis. The purpose of this research is to emphasize that pages are the basic unit of IoT name analysis that combines virtual and real. Focus on basic concepts, basic principles and basic methods, and typical cases (as 3 basic 1 example). Whether it is a form or chart compiled by an expert, or an audio and video recording (involving three-dimensional and living) taught by an expert, it can be included in the three basic and the examples, and it can be verified page by page. 


\subsection{Analysis - Can the quality of teaching continue to improve?}

- The first is that the "language point" is accurately in place. This is the jurisdiction of natural language understanding;

- Furthermore, the "knowledge points" are concise. This is the control range of expert knowledge expression;

- Finally, the source of the "original point" can be tested. This is the practical application of software pattern recognition.

The three points can be verified in time both online and offline and reused at any time (especially it can be called repeatedly in a targeted manner).

\subsection{Equations: Relational expression of data, information and knowledge}

The relationship between data, information and knowledge, in the jurisdiction of system engineering, not only has formal algebraic equations accurately expressed, but also has the balances of sequence and position for sequencing positioning identification analysis, and further, it can be connected with the code, card, table, library, network and terminal connection of the entire system and its back-end and front-end. Relational expression of data, information and knowledge, as in

$$
\mathrm{I}_{\mathrm{k}}+\mathrm{I}_{\mathrm{v}}=\mathrm{I}_{\delta} \square
$$

Equation (1) is the relational expression of or relationship between knowledge, information and data.

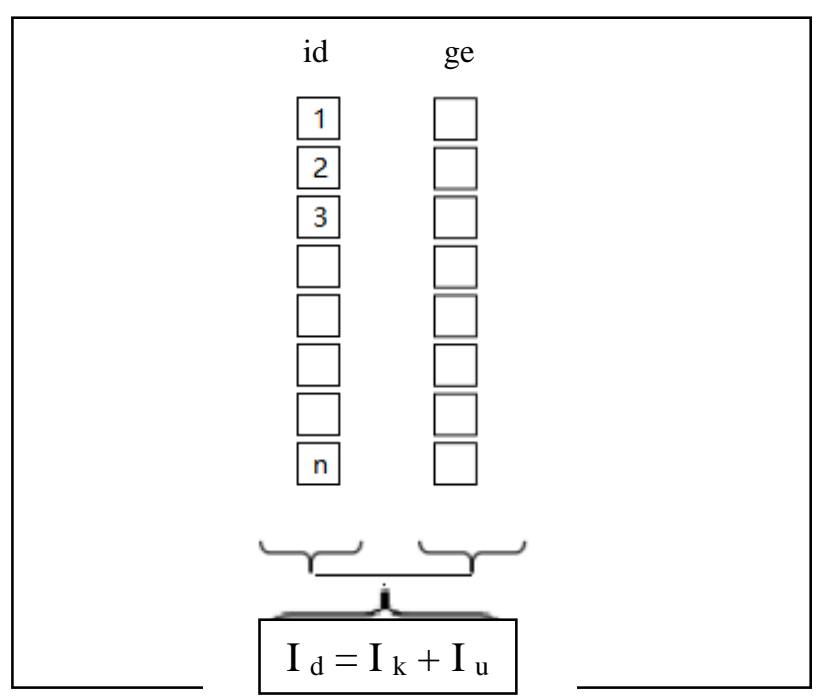

Figure 2. The balances of sequence and position, which can do sequencing positioning for each identification analysis point
It can be seen from Figure 2 that the double list $\{\mathrm{id}\}\{\mathrm{ge}\}$ is a twin Turing machine composed of numbers and spaces, based on the information identity $\mathrm{I}_{\mathrm{d}}=\mathrm{I}_{\mathrm{k}}+\mathrm{I}_{\mathrm{u}}$. The combination forms the model of formal understand. This is a system in which any type of symbolic form can be used to achieve indirect calculations.

\subsection{Discussion: Innovative management with the Internet of Things}

- First of all, on the basis of textbooks, courseware and high-quality courses by using recorded video, highlight the pages one by one as the basis of the Internet of Things names for the Educational Information (software pattern recognition) where the carrier and its name can be resolved.

- Furthermore, in terms of choosing intentions, we highlight the precise expression of three bases and one case (expert knowledge expression).

- Finally, in terms of language text, emphasize the completeness and accuracy of the knowledge system which consists of the term ontology (for the natural language understanding).

In the past, the content of textbooks, courseware and highquality courses almost all involved the copyrights of many people. As a result, it is difficult to trace the root cause of the "three points"- language point, knowledge point and original point. It is relatively easy to do within a single subject. It is quite difficult in large-scale multidisciplinary or interdisciplinary fields, in the field of complex subjects especially. On the one hand, interlacing is like a mountain, which leads to poor academic exchanges; on the other hand, there is no separation between lines for the integration of God' s wisdom, human and artificial intelligence can be achieved. However, because it significantly increases the cost of learning for teachers and students, so, it is difficult to achieve the collection of human knowledge.

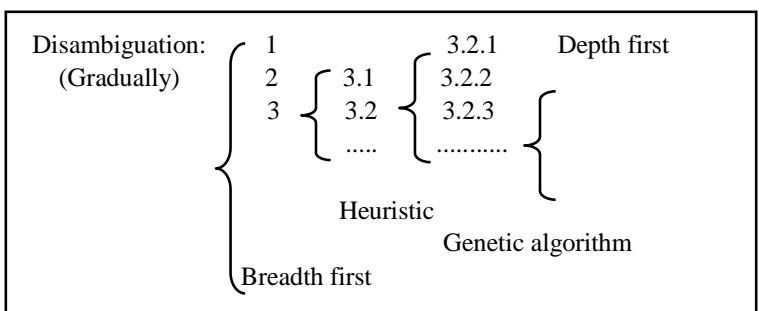

Figure 3. The number system (natural numbers and positive rational numbers) to resolve formal ambiguity step by step.

It can be seen from Figure 3 that natural numbers and positive rational numbers are expanded layer by layer to form an intuitive display of two types of basic search algorithms (breadth first and depth first). Its characteristic is to explain the search process of disambiguation step-by- 
step. In addition to breadth first and depth first, there are heuristics and genetic algorithms.

What is embodied here is the indirect formalized number, that is, the purely digital numbering system that can be resolved by the Internet of Things name.

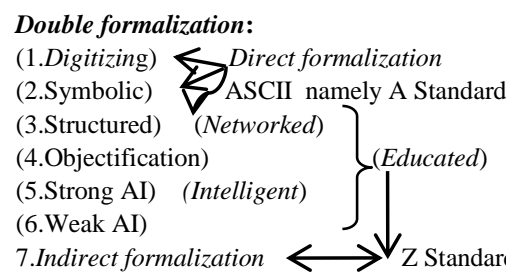

Figure 4. The development from A standard to Z standard has crossed several milestones

As can be seen from Figure 4, the digitizing, symbolization, and structuring standard for information processing in human-computer interaction is ASCII (abbreviated as A standard), which is essentially hexadecimal. The $\mathrm{Z}$ standard based on the $\mathrm{P}$ system not only has greater tolerance, but also has the characteristics of uniform precision and recall. The combination of decimal ID and network communication IP is the basic standard for contemporary IoT identification analysis.

U.S. and Chinese standards: Hexadecimal and P, both of that are compatible with man-machine standards: Binary and Decimal, which can achieve mutual conversion or form substitution in pure form.

Networking not only is to digitization, informationization, knowledge and intelligence as the "five modernizations", but also includes digitalization, symbolization, structuring, objectization, strong AI, weak AI,collaborative intelligence. The process of upgrading the formal technical standard of programming language development environment from A standard to $\mathrm{Z}$ standard accelerates the optimization process of human-machine collaborative intelligence.

It is characterized by the direct formalization of small character sets based on the A standard and the indirect formalization of large character sets based on the $\mathrm{Z}$ standard. Not only can they achieve the same results, but they can also come from behind, that is, the $\mathrm{Z}$ standard is compatible, supplements and covers A standard. Therefore, it has epoch-making revolutionary significance.

\section{CONCLUSION}

It is a basic work of international and domestic significance, that is conducive to the improvement and optimization of the "five modernizations" namely digitization, networking, information, knowledge and intelligence. The national Internet of Things names service platform Smart System Studied application promotion center characteristic model, paperless practice system and examination system can also be better popularized. The most important thing is that the advantages of human- machine collaborative intelligence are easier to form. This is a typical innovation management in educational technology. It understands the network-based education and training of teachers and students through the identification of the Internet of Things names (machineassisted by the first-level node and the second-level node).

\subsection{The advantages of human-machine collaborative intelligence are easier to form}

The core foundation of the human-machine collaborative smart system is the optimization of the formal standards for generalized bilingualism, generalized translation, and generalized language intention, and the key is humancomputer interaction. Example 1, American Standard Information Interchange Code (ASCII as A Standard), Example 2, China Standard Information Interchange Code, Example 3, Broad Bilingual Standard Information Interchange Code ( $\mathrm{Z}$ Standard). As far as this research is concerned, it is the eight major system projects that are connected through macro and micro. Based on them, the advantages of human-machine collaborative intelligence are easier to form.

\subsection{Typical Innovation Management in Educational Technology}

The reason why this is a typical innovation management in educational technology is not only because it understands the network-based education and training of teachers and students through the Internet of Things names service platform (first-level nodes and second-level nodes), but also because the combination of main and auxiliary lines reflects the overall advantages of "five innovations" and "five modernizations".

\section{ACKNOWLEDGMENT}

I would like to thank the Ministry of Education and various colleges and universities for providing analyzable examples for this research. Special thanks to the research team of Wang Xiaoqun of Peking University for hiring the author of this article, Xiaohui Zou, as a distinguished researcher for the corresponding interdisciplinary discussion in the past few years.

\section{REFERENCES}

[1] Ioannou, Andri and Chrystalla Antoniou. "Tabletops for Peace: Technology Enhanced Peacemaking in School Contexts.” J. Educ. Technol. Soc. 19 (2016): 164-176. 
[2] Marín, Victoria I., et al. "Open educational resources for research training: quality assurance through a collaborative evaluation." Research in Learning Technology 27 (2019): n. pag.

[3] Sekeroglu, et al. "Student Performance Prediction and Classification Using Machine Learning Algorithms." Proceedings of the Educational and Information Technology (2019): n. pag.

[4] Turcu, et al. "Blockchain and its Potential in Education.” ArXiv abs/1903.09300 (2019): n. pag..

[5] Asghari, Parvaneh, Amir Masoud Rahmani and Hamid Haj Seyyed Javadi. "Internet of Things applications: A systematic review" Comput. Networks 148 (2019): 241-261.
[6] Shunpeng Zou, Xiaohui Zou. "Understanding: How to Resolve Ambiguity.” IFIP TC12 ICIS (2017).

[7] Xiaohui Zou, Shunpeng Zou and Xiaoqun Wang. "The Strategy of Constructing an Interdisciplinary Knowledge Center.” ICNC-FSKD (2019).

[8] Chaurasia, A.. "Stop teaching Indians to copy and paste." Nature 534 (2016): 591-591. 